\title{
A Co-operative Hybrid Algorithm for Fault Diagnosis in Power Transmission
}

\author{
Ramesh K. Rayudu, Member, IEEE Sandhya Samarasinghe, Member, IEEE

\begin{abstract}
Transpower AI Research Lab, NRE Group, Lincoln University, Canterbury, New Zealand. Email: Ramesh.Rayudu@transpower.co.nz
\end{abstract}

Ajay Maharaj

\begin{abstract}
This paper presents our co-operative hybrid algorithm for fault diagnosis in power transmission networks. When a fault occurs in a transmission network, it must be identified and eliminated as soon as possible. Since control centers are flooded with hundreds of alarm messages during a fault, fault diagnosis, which involves the analysis of alarm messages, is a time consuming task. Towards the development of a fault diagnostician, model-based, heuristic, and neural networks are applied to the domain and the results are presented in this paper. The algorithm is a hierarchical model which combines several reasoning methods such as heuristic, temporal and model-based diagnosis and incorporates a network of neural networks at one of the levels of the hierarchy. The working of this co-operative algorithm is discussed and its results are analysed.
\end{abstract}

Keywords: Intelligent Systems, Intelligent Fault Diagnosis; Power Transmission Networks, Hybrid Algorithms; Transpower Investigations, Model based Diagnosis, Co-operative systems

\section{INTRODUCTION}

Power transmission network fault diagnosis (PTNFD) is the process of detecting faults while it is in operation. This kind of diagnosis (also termed as operative diagnosis) is needed for systems which cannot be stopped for maintenance (as it is too expensive), and the diagnosis involves the consideration of symptoms and state which can change with time. In electrical power transmission networks, the diagnosis is confined to alarm readings in real time while the effects of the faults are still propagating through the network. PTNFD is heuristic in nature and often provides a challenging task for experts involved. Experts find that the pattern recognition of alarms triggered by a fault in the system is relatively easier task compared to the identification of the physical origins of the fault from a list of alarms. This difficulty could be due to several components malfunctioning at the same time within the network.

Power transmission networks carry power from supply utilities to the consumer and any fault in the network directly affects the consumers. The hazards of performing fault diagnosis in this domain incorrectly and too slowly result in notable accidents such as 1977 New York City blackout where the power restoration took several minutes causing inconvenience to consumers. Earlier research [2] has shown that decision-support systems can aid system controllers during emergency situations. This paper presents our algorithm for an efficient fault diagnostician for power transmission network in New Zealand. Towards this development, model-based, heuristic and neural network techniques are applied to the domain and the results are presented in this paper.

\section{MODEL BASED DIAGNOSIS}

Model based diagnosis is suitable for power transmission fault diagnosis for many reasons including the following:

Protective relay systems, which are the main indicators of the power system status, can be modeled as discrete-event systems where each component will have some discrete states. Every component in the network will interact with each other in an event and alter the states of related components accordingly. It is this inter-related concept which makes MBD suitable for power system fault diagnosis.

$>\mathrm{MBD}$ covers a wide range of fault scenarios than heuristic reasoning because MBD is based on the behavioral analysis of the system.

> MBD can detect deviations from the expected behavior.

$>$ MBD can detect malfunctioning equipment in the early stages.

$>\mathrm{MBD}$ can predict the effects of the faults and unnecessary alarms as it simulates the faults.

$>\mathrm{MBD}$ can handle multiple faults efficiently because the cascading effects of the faults can be simulated and analyzed.

$>$ MBD can adopt to goal driven reasoning of rule-based systems [10].

Eventhough model based diagnosis provides a promising solution to power transmission fault diagnosis, it suffers from several limitations.

- MBD is suitable for systems that can be modeled easily [10]. Since power networks involve several components and complex protective behavior, modeling of power networks is a time-consuming task.

- Since MBD systems work on generate-and-test reasoning, it takes more time to arrive at a solution.

- Since power networks change its topology over time, the online functional modeling of a wide network is time consuming which adds further delay to MBD.

To overcome some of these difficulties, we attempt to represent and diagnose the problem at different levels of power network configuration by combining both model-based 
and heuristic reasoning techniques. These attempts are discussed and the resulting algorithm is described in the following sections.

\section{HIERARCHICAL HEURISTIC-MODEL-BASED \\ FAULT ANALYSIS AND DIAGNOSIS (HIMOBFAD) IN POWER TRANSMISSION NETWORKS}

HiMoBFAD is a heuristic-model-based hybrid design for diagnosis of continuous-time dynamic operative systems. The algorithm's structure is primarily based on Mozetic's hierarchical model based diagnosis [1] where a diagnosis is performed at different hierarchies of the algorithm. Figure 1 presents an abstract view of HiMOBFAD's architecture in which three tasks mediate between the real-world system through SCADA and its models in HiMoBFAD. The three tasks as shown in the figure are summarized as follows:

1. Actions: The purpose of actions task is to inform the operator of the current and final states of a diagnosis.

2. Diagnosis: The purpose of diagnosis task is to analyze and identify the cause of faults. In HiMoBFAD, it is done in two phases: the pre-diagnosis and final diagnosis. Prediagnosis uses relevant component models to model the behavior of protection and status equipment. The observed behavior is compared with the expected behavior of the models and conclusions about the correctness of components are drawn from the comparison. The purpose of final diagnosis phase is to identify faults and details of the faults and determine where and why the fault has occurred. This phase uses the diagnostic output from pre-diagnosis phase.

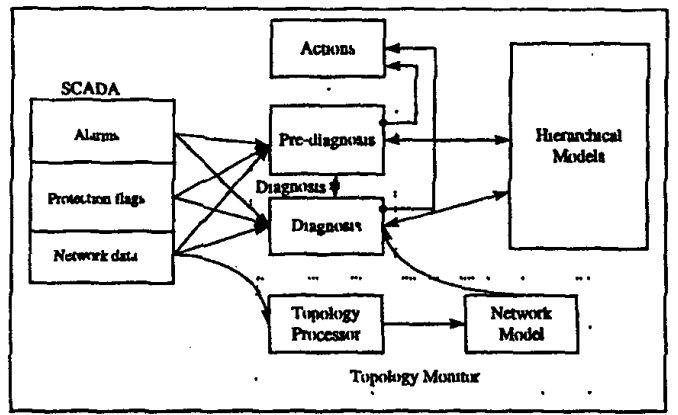

Figure 1. Design of HiMoBFAD's architecture.

3. Topology monitoring: The purpose of this task is to update the state of the model to the real-world power system's observed state and to provide the direction of power-flow between substations in the network. This task also helps to check the consistency of incoming alarms with respect to current state of systems.

\section{A. Modeling in HiMoBFAD}

Power transmission network is a network of components essential for transmitting the power between two stations. To develop a model of the network one must represent all the components, their relationships and behaviors in the model. HiMoBFAD's power network model is represented in two distinct models: a structural model representing components and their connections, and a functional model that predicts possible behaviors given a fault hypothesis. This section describes the structural and functional model representations of HiMOBFAD. As stated in the beginning of section 3, HiMoBFAD's representation is primarily based on Mozetic's hierarchical model based diagnosis [1] but differs from it in several aspects and are stated in [13].

\section{B. Network Structural Model}

The structural model of HiMoBFAD is described in two types of hierarchies:

1. Power network's topological hierarchy where the network is divided into a four level hierarchy based on the topology of the network

2. Object oriented class hierarchy where the power network components are arranged in a three level hierarchy using object oriented programming techniques.

\section{B.1. Power network topological hierarchy}

Power transmission network is a network of components essential for transmitting the power between two stations. To model the components and their topological relationships we have divided the power network into four topological levels of hierarchy. Figure 2 shows the hierarchy of HiMoBFAD. The first level (level 1) contains all the components in the network such as circuit breakers, protective relays, lines, feeders, busbars, transformers, generators, etc. The connection between all the components represented in level 1 are represented hierarchically from level 2 to 4 (Figure 2). Level 2 consists of data relating to switching groups, which are connections between circuit breaker and isolators. Level 3 represents the connection of two switching groups and the operational equipment between them such as a transmission line or a transformer. These connections at level 3 are called clusters. The top level (level 4) represents the connections between clusters that make the complete power network. 


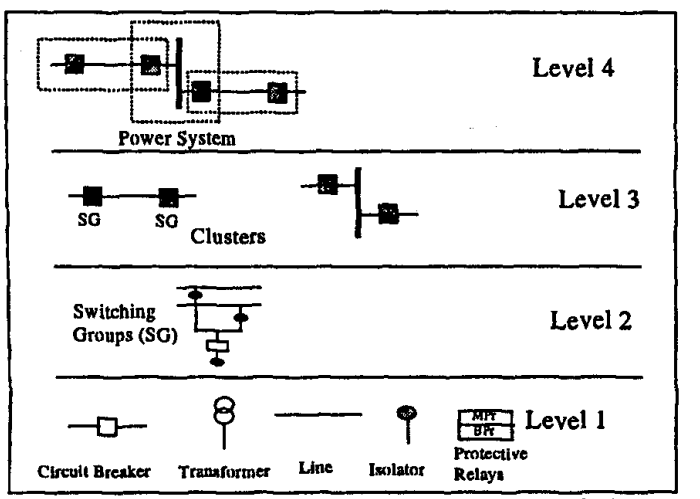

Figure 2. Hierarchical topological network representation of HiMoBFAD.

\section{B.2. Object oriented $(O O)$ class hierarchy}

The application of $\mathrm{OO}$ representation mainly consists of three subjects: objects, messages and classes. Objects are entities that encapsulate a data set and can only be accessed or modified by activating the resident methods. The method activation is done by passing messages requesting the object to either provide access to or to modify a specified data. Each object instantiates a certain class. The classes can be organized hierarchically such that some of the attributes implemented in upper-levels can be recognized by lower levels. This process of recognition is called inheritance.

A typical transmission network consists of customer points, transformers, switching equipment such as circuit breakers and isolators, bus-bars, lines, feeders, condensers and protection equipment. Based on the concepts and principles presented for $O O$ representation above, a transmission system is modeled as a collection of objects. Each object is an instance of the corresponding component type represented as a class. Each component class is defined as a generic component with several qualitative states as attributes. For example, the circuit breakers, isolators and protection equipment can be treated as status equipment (SE) and $O O$ representation for SEs will have 'status equipment' as Super-class and circuit breakers, isolators and protection equipment as its subclasses. The representation is pictorially represented in Figure 3.

\section{Network Functional Model}

The functional modeling of HiMoBFAD is based on the functional relationships between the components specific to power transmission networks. It incorporates the behavioral knowledge of the components. The behavioral knowledge of all the components is based on different protection and component operation models and is derived from their modelbased knowledge. As in HiMoBFAD's structural modeling, the functional modeling is also represented in four levels.

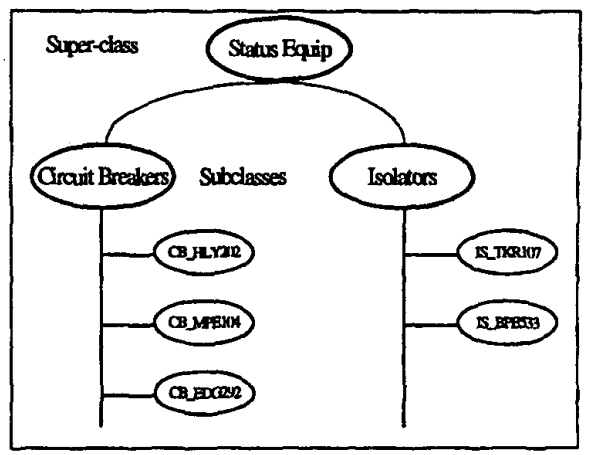

Figure 3. Object oriented relationship between 'status equipment' components.

The behavioral knowledge related to circuit breakers, isolators and protection equipment is incorporated into their respective models in 'Level 1' of the hierarchy. For example, consider a typical network shown in the Figure 4. The 'Level 1 ' represents behavioral models of individual components such as CB1, CB2, CB3, and their present status (either open or closed). The connections between protective relays, circuit breakers, and isolators denote switching groups and the behavioral models related are represented in the second level (Figure 5). These models are also derived from model based knowledge.

The representation of entities bounded by a set of switching groups (also called clusters) make the third level. The clusters are similar to relay protection zones identified by the protection engineers for ground-fault isolation. Hence the cluster level incorporates behavior knowledge resembling the behavior of two connected switching-groups and the operational equipment between them (transformer, line, etc.). The behavior of each cluster is modeled as a neural network and is used as a 'network of neural nets' at this level. The architecture of this level with neural nets is discussed in Rayudu \& Samarasinghe [14]. The behavioral knowledge of this level is derived from domain's model-based knowledge and the problem solving knowledge incorporates the heuristic knowledge of the cluster. At this level the diagnosis is done to check the behavior of components associated with the cluster. Figure 6 shows the clusters in our example network. The fourth level, which is also the final level of the functional hierarchy, represents the whole network in terms of clusters. This level does not incorporate any behavioral knowledge because the behavior-check of all the components is already done in the lower levels. This level encompasses only the strategic problem solving heuristic knowledge related to the complete power network. The diagnostic output from Level 3 is analyzed in this level to identify the cause of fault. 

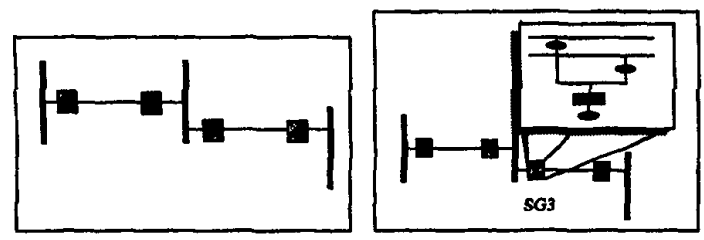

Figure 4 \& 5. Example Network and Switching Groups in the network.

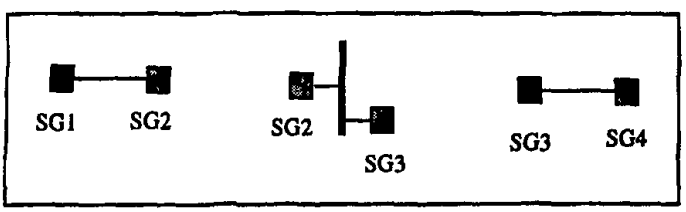

Figure 6. Cluster formats in the network.

The diagnostic algorithm of HiMoBFAD is similar to Pfau-Wagenbauer et.al [6]. The algorithm is a combination of model-based and heuristic knowledge. In general, the diagnostic algorithm of HiMOBFAD incorporates diagnostic procedures followed by HiMoBFAD. These procedures include:

1. reasoning mechanisms such as truth maintenance, backward chaining, forward chaining and causal reasoning

2. search mechanisms such as heuristic and depth-first search

3. transitional constraints between different levels of HiMoBFAD

4. conflict resolution (multiple solutions and multiple faults)

5. procedure to analyze circuit breaker failure protection

Level 1:

$o k(C B) \Leftrightarrow \operatorname{tripped}(C B)$ or

operated(MPr or $B P r$ ) and open( $C B)$

$o k(M P r) \Leftarrow \operatorname{operated}(M P r)$ and open $(C B)$

Level 2:

$o k\left(M P r_{-}\right.$relay $) \Leftarrow o k\left(M P r_{-} X\right) \& o k\left(M P r_{-} Y\right)$

$o k\left(S G \_X\right) \Leftarrow(o k(M P r)$ or ok $(B P r)) \&$ ok $(C B)$

Level 3:

$\operatorname{linefault}(L X) \Leftarrow o k\left(S G \_X\right) \& o k\left(S G \_X 2\right)$

Level 4:

cable_fault $\Leftarrow\left(o k\left(M P r_{-}\right.\right.$relay) \& operated(EF)) or

(pilotfault $\left(S \bar{G} \_X\right)$ \& operated $(E F)$ )

Figure 7. Example rules in HiMoBFAD.

\section{GOAL DRIVEN REASONING IN HIMOBFAD}

Let us consider a simple example network shown in the Figure 8. It shows the breaker trip situation after a fault on line line2. Given this training example, the task of HiMOBFAD is to determine which of the components are relevant to the goal concept. The fault in Figure 8 raises alarms which are injected into level 1 of HiMoBFAD to replicate the current situation of the network. At this level itself, the components are checked for their authenticity of operation. This is done by the qualitative rules shown in the Figure 7. For this example, all the operations were genuine. Once the authenticity is proved the rules at level 2 (switching groups) will operate to check the genuine operation of switching groups. At this level it proves that the switching groups at $S G 1, S G 2, S G 3$, and $S G 4$ have operated correctly. From switching groups, the analysis moves on to cluster level (level 3). At this level, preliminary diagnosis is made where the rules are applied on each affected cluster. At this level, it is diagnosed that the fault is in cluster 2 as both the circuit breakers on the either side of line 2 operated but no diagnosis is reached on cluster 1 as only one breaker operated. These inferences will be sent to level 4 (power system level) to deduce the fault. In this level, the relation between two clusters is checked to find the relationship between two diagnoses. For this example, the relationship between the two clusters is established and at the first instance, it is inferred that the two diagnoses are inter-related. Then the relationship is established by a resolution algorithm which finally provides the main cause of failure.

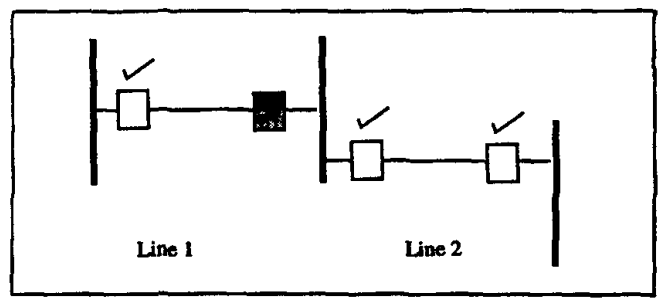

Figure 8. Breaker trip situation in the network shown in Figure 4.

Depending upon the problem, HiMoBFAD takes 0.13 to 5.8 seconds to arrive at a diagnosis.

It is important to note that HiMoBFAD does not use continuous variables such as voltages and power flows in its diagnostic process. Some of the important reasons for this are given below:

- Symbolic reasoning or learning systems seldom use continuous variables and often it is possible to elicit most or all of the useful information from a continuous variable by applying a Boolean test, e.g. " $X<=5.6$ ".

- It has been proven by several researchers [12] that inclusion of continuous variables did not significantly improve the amount of information that could be elicited from the breaker openings and network islands.

- In symbolic reasoning systems, computational efficiency will be maintained if continuous variables are not used. Unless the reasoning system can inherently handle continuous variables (e.g. neural networks), the introduction of continuous measurements will slow the process. 


\section{CONCLUSION}

We have presented a hybrid algorithm applied to power transmission fault diagnosis based on model-based reasoning and heuristic reasoning. HiMoBFAD, a heuristic-model based system, is based on Igor Mozetic's Hierarchical Model Based Diagnosis and incorporates the domain models in four levels; the third and fourth levels being heuristic levels. The hybrid algorithm, HiMoBFAD, works faster than traditional model based diagnosis [13] but takes more time than heuristic based diagnosis [13]. HiMoBFAD can solve most diagnostic problems in power transmission networks and can handle multiple fault scenarios and multiple solutions. The system is currently under full implementation for Transpower NZ Limited.

\section{ACKNOWLEDGMENTS}

We thank Mr. Kevin Mackey, General Manager (SD), and Mr. Mark Pearce, Grid Operating Services Manager of Transpower NZ Ltd. for their support and Control Centre Staff at NCC, Trans Power NZ Ltd. for their feedback and support.

We also thank Dr. Eduardo Morales of ITESM, Mexico, Dr. Rambabu Adapa of EPRI, USA and Dr. John Ypsilantis of Heuristic Australia Ltd. for their support and suggestions.

\section{REFERENCES}

[1] Mozetic I., "Hierarchical Model-Based Diagnosis", Intl. Jnl. Man-Machine Studies, 35 (3), 1991, 329362.

[2] Wollenberg B. F., "Feasibility study for an Energy Management System Intelligent Alarm Processor", IEEE Trans. On Power Systems, May 1986, pp. 241247.

[3] Reiter R., "A Theory of Diagnosis from First Principles", Artificial Intelligence 32, 1987, pp. 5795.

[4] Johan de Kleer \& Williams B. C., "Diagnosing Multiple Faults", Artificial Intelligence 32, 1987, pp. 97-130.

[5] Mozetic I., "Diagnostic efficiency of deep and surface knowledge in KARDIO", AI in Medicine 2 (2), 1990, pp. 67-83.

[6] Pfau-Wagenbauer M., and Nejdl W., "Integrating Model-based and heuristic features in a real-time Expert System", IEEE-EXPERT, August 1993, pp. 12-18.

[7] El Fattah Y. \& O'Rorke P., "Explanation-Based Learning for Diagnosis", Machine Learning, 13,1993, pp. 35-70.

[8] Clark P. \& Holte R., "Lazy Partial Evaluation: Ar integration of explanation based generalisation anc partial evaluation", Proc. 6th European Conf. $\mathrm{O}_{1}$ Machine Learning (ECML-93), 1993, pp. 360-365.
Mitchell T. M., Keller R. M., and Kedar-Cabelli S. T., "Explanation based generalisation: A unifying view", Machine Learning, 1, 1987, pp. 47-80.

0] Wang X., Dillon T., "A second generation expert system for fault diagnosis", Electrical Power \& Energy Systems, Vol. 14, No 2/3, April/June 1992, pp. 212-216.

11] Rayudu R.K., Samarasinghe S., Kulasiri D., "A Comparison of Model-based Reasoning and Learning approaches to Power Transmission Fault Diagnosis", 2nd Intl. Two-stream conference on Artificial Neural Networks and Expert Systems ANNES-95, Dunedin, New Zealand, 1995, pp. 218-222.

[12] Ypsilantis J., Yee H., \& Teo C. Y., "Adaptive rule based fault diagnostician for power distribution networks", IEE Proc. Generation, Transmission and Distribution, V 139, Nov 1992, pp. 461-468.

[13] Rayudu R.K. and Samarasinghe S., "Integration of Model-based and heuristic fault diagnosis in power transmission network", IPENZ Engineering Conference, Dunedin, 1996, Vol. 2, No1, pp. 112118.

[14] Rayudu R.K. and Samarasinghe S., "A Network of Neural Nets to model power system networks for fault diagnosis", Progress in Connectionist based Information Systems, Vol. 2, Springer-Verlag Singapore, pp. 1004-1008.

\section{BIOGRAPHIES}

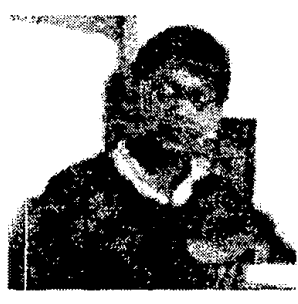

Ramesh Rayudu graduated in Mechanical Engineering from Osmania University, India in 1989. He then worked for a Company as a Project Engineer until 199 land as Senior Technical Executive for a Software Company in Hyderabad, India. In 1992 he left for New Zealand to study $\mathrm{ME}$ in Control Engineering in University of Canterbury.

After completing his ME in $1993 \mathrm{He}$ joined Lincoln University to work on a project for Transpower NZ Ltd. Since then he worked for Transpower on several projects. Presently he is working on a smart fault diagnostician for Transpower Networks. His research interests include Intelligent A.utomation, Soft-computing applications, Intelligent Control systems, Model-based diagnosis, Human behavior modeling and Machine learning.

Ramesh has written several invited articles for magazines and conferences and has won 3 Best Presentation awards. This year he has been awarded 1999 Fulton-Downer Silver Medal Award for Best Paper by IPENZ, New Zealand.

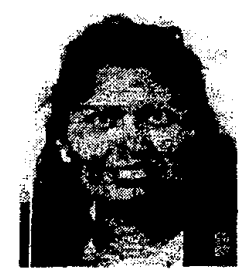

Sandhya Samarasinghe received her MS and $\mathrm{PhD}$ in Engineering from Virginia Polytechnic Institute and State University (VPI \& SU), Virginia, USA in 1987 and 1991 respectively. She is currently a lecturer in Natural Resources Engineering Group at Lincoln University, Canterbury, New Zealand. 
Her research interests include computing and image processing applications in engineering and forest industries, and AI applications in engineering.

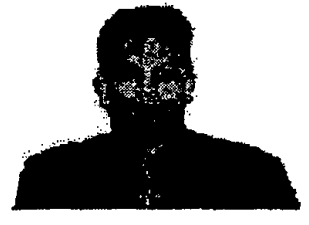

Ajay Maharaj is the National Coordination Centre Manager for Transpower NZ Ldd., and leads a team that manages Transpower's high voltage network in real-time. This team also delivers services to the Wholesale Electricity Market of New Zealand. He completed his NZCE (Mechanical) before joining the electricity industry.

Since then he holds a Diploma in Manngement Studies and Masters in Business Administration (MBA) degrees from the University of Waikato, New Zealand. 